\title{
PERCEPÇÃO DOS DÉFICITS COGNITIVOS E ALTERAÇ̃̃ES DO COMPORTAMENTO EM PACIENTES COM DOENÇA DE ALZHEIMER
}

\author{
OSVALDOP.ALMEIDA*, ELISETEI. CROCCO*
}

\begin{abstract}
RESUMO - A doença de Alzheimer (DA) está associada a deterioração das habilidades intelectuais e, com frequência, do comportamento do paciente. O paciente, porém, tem percepção limitada da gravidade e qualidade dessas alterações. Nós desenhamos este estudo para investigar a concordância entre pacientes e cuidadores na avaliação de dificuldades cognitivas e alterações do comportamento associadas à DA. Trinta pacientes com diagnóstico de DA (DSM-IV) atendidos consecutivamente no ambulatório de saúde mental da Santa Casa de São Paulo foram recrutados para inclusão no estudo. Um cuidador foi também selecionado para cada paciente. A concordância entre pacientes e cuidadores quanto às dificuldades cognitivas e alterações comportamentais foi avaliada através da versão ampliada do questionário para demência-anosognosia (QD). As habilidades cognitivas de pacientes e cuidadores foi avaliada através do mini exame do estado mental (MMSE). A idade média dos pacientes e cuidadores era 71,38 (IC=68,23 a 74,53) e 52,48 anos (IC=47,11 a 57,86) respectivamente. Sessenta por cento e $73,3 \%$ dos pacientes e cuidadores eram do sexo feminino. O escore médio dos pacientes no MMSE foi 14,93 (IC=12,68 a 17,18). A concordância entre pacientes e cuidadores para os escores de itens individuais do QD, de acordo com o índice Kappa ponderado, variou de 0 a 0,67, embora valores menores do que 0,40 fossem observados para 39 dos 42 itens. O escore total dos pacientes na seção do QD que avalia habilidades cognitivas (QD-A) foi significativamente menor do que para os cuidadores (t-pareado $=-4,07, \mathrm{p}<0,001$ ). $\mathrm{O}$ mesmo padrão de resposta foi observado na seção do questionário que avalia comportamento (QD-B).(t-pareado= -2,27, $\mathrm{p}=0,032$ ). A correlação de Spearman entre os escores do QD-A e MMSE de acordo com o paciente e cuidador foi $-0,39$ e $-0,57$ respectivamente. Anosognosia cognitiva (diferença entre o QD-A de cuidadores e pacientes) não se correlacionou de forma significativa com o escore do MMSE $($ rho $=-0,14)$ ou presença de depressão entre os pacientes $(t=-0,40, p=0,698)$. Estes resultados indicam que os pacientes têm percepção limitada dos déficits cognitivos e alterações de comportamento associadas à DA. Além disso, eles sugerem que a baixa auto-crítica dos pacientes não é influenciada de forma importante pela gravidade do quadro demencial ou presença de sintomas depressivos.
\end{abstract}

PALAVRAS-CHAVE: demência, doença de Alzheimer, anosognosia, insight, crítica, auto-percepção, diagnóstico, cuidador, MMSE, déficits cognitivos, memória, transtornos do comportamento, sintomas psiquiátricos.

Awareness of cognitive deficits and behavioural abnormalities amongst patients with Alzheimer's disease

ABSTRACT - Alzheimer's disease (AD) is associated with cognitive decline and, often, the development of behavioural abnormalities. However, patients may have limited insight into the severity and extent of their impairments. This study was designed to investigate agreement between patients and carers on the assessment of cognitive and behavioural symptoms typically associated with AD. Thirty consecutive outpatients meeting criteria for the diagnosis of AD according to DSM-IV and their respective carers were invited to participate in this study. An enlarged version of the questionnaire for dementia-anosognosia (QD) was used to assess cognitive and behavioural symptoms according to patients and carers. Cognitive impairment was further assessed with the mini-mental state examination (MMSE). The mean age of patients and carers was $71.38(\mathrm{CI}=68.23$ to 74.53$)$ and $52.48(\mathrm{CI}=47.11$ to 57.86 ) respectively. Sixty and $73.3 \%$ of patients and carers were women. The mean MMSE score for patients was 14.93 ( $\mathrm{CI}=12.68$ to 17.18 ). Agreement between patients and carers for all QD items was assessed using weighted Kappa - rates were low and ranged from 0 to 0.67 . Only 3 of the 42 QD items were associated with Kappa values greater than 0.40 . The overall score for questions assessing cognitive abilities (QD-A) was significantly lower

*Estudo realizado no Departamento de Saúde Mental da Santa Casa de São Paulo. Elisete I Crocco foi parcialmente financiada por bolsa de iniciação científica do CNPq. Aceite: 3-fevereiro-2000.

Dr. Osvaldo P. Almeida, UWA Department of Psychiatry, Queen Elisabeth II Medical Centre, Nedlands, Perth, WA 6009, Australia. Fax 6189346 3828. Email: osvalm@cyllene.uwa.edu.au 
according to the evaluation of patients than that of carers (paired t-test $=-4.07, \mathrm{p}<0.001$ ). The same pattern was observed for questions assessing behaviour (QD-B)(paired $\mathrm{t}$-test $=-2.27, \mathrm{p}=0.032$ ). The Spearman correlation between QD-A and MMSE scores was -0.39 and -0.57 according patients and carers respectively. The association between cognitive anosognosia (difference between QD-A according to carers and patients) and MMSE scores was poor $($ rho $=-0.14)$. In addition, cognitive anosognosia scores of patients with and without significant depressive symptoms was similar $(\mathrm{t}=-0.40, \mathrm{p}=0.698)$. These results suggest that $\mathrm{AD}$ patients have limited insight into the severity and extent of their cognitive and behavioural problems. In addition, our data shows that this lack of awareness is not influenced by the severity of cognitive impairment or the presence of depressive symptoms.

KEY WORDS: Dementia, Alzheimer's disease, anosognosia, awareness, insight, diagnosis, assessment, cognition, MMSE, cognitive decline, memory impairment, behavioural symptoms, behavioural abnormalities, psychiatric symptoms.

O envelhecimento da população mundial durante as últimas décadas fez com que a demência passasse a ser um dos mais importantes problemas de saúde pública da atualidade ${ }^{1,2}$. A doença de Alzheimer (DA) é a causa mais frequente de demência ${ }^{3}$ e seu diagnóstico clínico depende da demonstração da existência de declínio em habilidades intelectuais como a memória, linguagem, percepção, atividades motoras, abstração e planejamento ${ }^{4}$. Assim sendo, o diagnóstico de demência e da DA passou a ter como base a investigação da capacidade cognitiva de populações de risco com instrumentos como o mini exame do estado mental (MMSE) ${ }^{5}$ e similares. Uma forma alternativa de investigar a presença de demência é através de entrevista com o cuidador do paciente. Jorm ${ }^{6}$ reviu as características de quatro instrumentos utilizados para essa finalidade e concluiu que eles são confiáveis, apresentam alta correlação com avaliações cognitivas do paciente e são capazes de discrimiar adequadamente indivíduos com e sem demência.

A avaliação de pacientes com demência não termina, porém, com a demonstração de que dificuldades cognitivas estão presentes. Alterações do comportamento podem ser observadas em praticamente metade desses indivíduos em estudos transversais ${ }^{7}$, embora a detecção de vários desses sintomas dependa, com frequência, de informações fornecidas pelo cuidador do paciente. $\mathrm{O}$ estudo de Burns et al. ${ }^{8}$ é um exemplo das dificuldades e discrepâncias que podem ocorrer durante a avaliação desses sintomas. Eles investigaram a presença de distúrbios do humor em 178 idosos vivendo na região sudeste de Londres. O examinador observou sintomas depressivos em 23,5\% dos casos esse resultado foi sensivelmente diferente das taxas de $42,9 \%$ e $63,0 \%$ descritas por cuidadores e pacientes respectivamente.

Nós desenhamos o presente estudo com o objetivo de investigar a concordância entre pacientes e cuidadores na avaliação das dificuldades cognitivas e comportamentais associadas à DA. Além disso, procuramos esclarecer se a gravidade da demência e a presença de sintomas depressivos contribuem para reduzir a percepção crítica do paciente em relação a seus déficits.

\section{MÉTODO}

Pacientes com DA atentidos consecutivamente no ambulatório de saúde mental da Santa Casa de São Paulo entre outubro de 1997 e junho de 1999 foram convidados a participar do estudo. O diagnóstico de doença de Alzheimer foi feito de acordo com os critérios da Associação Americana de Psiquiatria9. Para cada paciente foi selecionado um cuidador (não remunerado). O cuidador foi definido como: (1) indivíduo responsável pelo cuidado do paciente em suas atividades rotineiras (como por exemplo, finanças) e, (2) contato direto com o paciente de pelo menos 4 dias por semana. Os objetivos da pesquisa foram, então, relatados aos pacientes e seus cuidadores — a inclusão no estudo ocorreu apenas após o consentimento informado de ambos.

A coleta de dados incluiu:

1. Pesquisa de variáveis sócio-demográficas básicas (idade, sexo, estado civil, escolaridade e renda familiar média)

2. Mini-Exame do Estado Mental - MMSE ${ }^{10}$. O MMSE avalia orientação temporal e para espacial, memória imediata, cálculo, memória recente, linguagem e apraxia construtiva. A escala foi traduzida e validada para uso em nosso meio ${ }^{5}$. Os escores variam entre 0 e 30 pontos - MMSE $\geq 18$ indica a presença de demência de gravidade leve, $10 \leq \mathrm{MMSE}<18$ demência com gravidade moderada, e MMSE $<10$ demência grave. 
3. Questionário de Demência-ampliado (QD). É composto por 42 questões divididas em duas seções. A primeira (seção A) avalia funcionamento intelectual (por exemplo: "Você tem problemas para se lembrar de datas?"), enquanto a segunda (seção B) avalia alterações do comportamento (por exemplo: "Você se sente mais deprimido?'). Cada uma das questões recebe um escore: nunca (0), algumas vezes (1), com frequência (2), e sempre (3). As questões são apresentadas em uma versão para o paciente e outra para o cuidador. O QD inclui todos os 30 itens utilizados por Migliorelli e et al. ${ }^{11}$ para avaliar a presença de anosognosia (traduzidos do inglês para o português e novamente de volta para o inglês a fim de garantir a precisão da tradução): QD-A itens 1 a 22 e QD-B ítens 1 a 8. Além disso, ítens suplementares foram incluídos para avaliar funções básicas da vida diária e permitir avaliação mais extensa de alterações do comportamento (Tabelas 2 e 3). Para efeito deste estudo, anosognosia cognitiva e comportamental foram definidas como a diferença entre os escores de cuidadores e pacientes nas seções A e B do QD respectivamente.

A avaliação dos pacientes seguiu sempre a mesma estrutura: eles eram entrevistados para coleta das informações sócio-demográficas, completavam o MMSE e, finalmente, respondiam às questões do QD. O mesmo procedimento foi utilizado para avaliação dos cuidadores. Todas as avaliações foram realizadas por um dos autores (EIC).

Os dados deste estudo foram analisados através do programa estatístico 'Stata, release 6.0' ${ }^{12}$. Análise de probabilidade de tabelas de contingências foi utilizada na investigação de informações de variáveis categoriais, o resultado estatístico sendo distribuído como qui-quadrado de Pearson $\left(\chi^{2}\right)$. A concordância entre os escores no QD foi estimada através do teste estatístico Kappa ponderado. O teste t-pareado foi utilizado para comparar diferenças entre os escores do paciente e seu respectivo cuidador (por exemplo, escore total no QD), enquanto o teste 't de Student' foi utilizado para comparar escore de dois grupos (por exemplo, escores do MMSE para pacientes com e sem depressão). A correlação não-paramétrica de Spearman foi utilizada para verificar a associação entre os escores (por exemplo, QD e MMSE). Intervalos de confiança de 95\% (CI) foram calculados para a média de variáveis como idade e escores do MMSE e QD.

\section{RESULTADOS}

Trinta pacientes e cuidadores foram recrutados para participarem do estudo. Não houve recusas. A Tabela 1 resume as características sócio-demográficas básicas da amostra. A idade dos pacientes

Tabela 1. Características sócio-demográficas de pacientes com doença de Alzheimer e de seus respectivos cuidadores.

\begin{tabular}{|c|c|c|c|c|}
\hline & Pacientes $(n=30)$ & Cuidadores $(n=30)$ & $\mathrm{t}$-pareado, $\chi^{2}$ & $\mathrm{p}$ \\
\hline Idade média*1 & 71,38 & 52,48 & 6,04 & $<0,001$ \\
\hline $\mathrm{IC}=$ & 68,23 a 74,53 & 47,11 a 57,86 & & \\
\hline Sexo feminino $(\%)$ & 60,0 & 73,3 & 3,44 & 0,064 \\
\hline Estado civil (\%) & & & 4,02 & 0,910 \\
\hline solteiro & 16,7 & 23,3 & & \\
\hline casado & 46,7 & 56,7 & & \\
\hline separado/divordicado & 6,7 & 10,0 & & \\
\hline viúvo & 30,0 & 10,0 & & \\
\hline Escolariedade $(\%)$ & & & 11,02 & 0,527 \\
\hline Nunca frequentou & 23,3 & 0 & & \\
\hline primário & 40,0 & 23,3 & & \\
\hline ginásio & 13,3 & 23,3 & & \\
\hline colegial/normal & 16,7 & 30,0 & & \\
\hline terceiro grau & 6,7 & 23,3 & & \\
\hline $\begin{array}{l}\text { Renda familiar média } \\
(\text { (em salários mínimos)*5 }\end{array}$ & 6,50 & 7,85 & $-2,15$ & 0,042 \\
\hline $\mathrm{IC}=$ & 3.29 a 9,71 & 4,74 a 10,96 & & \\
\hline Escore médio no MMSE*1 & 14,93 & 27,14 & $-10,46$ & $<0,001$ \\
\hline $\mathrm{IC}=$ & 12,68 a 17,18 & 26,32 a 27,95 & & \\
\hline
\end{tabular}

IC, intervalo de confiança de $95 \%$ da média; *n, número de pares para os quais a informação não estava disponível. 
Tabela 2. Concordância dos escores entre pacientes e cuidadores para os itens do questionário de demênciaseção A: funções intelectuais e atividades rotineiras

\begin{tabular}{|c|c|c|c|c|c|}
\hline Item do Questionário & $\begin{array}{l}\text { \% de pacientes } \\
\text { com escore } \\
\text { menor que o } \\
\text { do cuidador }\end{array}$ & $\begin{array}{c}\text { Concordânica } \\
\text { observada } \\
(\%)\end{array}$ & $\begin{array}{c}\text { Concordância } \\
\text { esperada } \\
(\%)\end{array}$ & $\begin{array}{l}\text { Kappa } \\
\text { ponderado }\end{array}$ & $\mathrm{p}$ \\
\hline 1. dificuldades para se lembrar de datas & 53,3 & 62,2 & 64,6 & $-0,07$ & 0,723 \\
\hline $\begin{array}{l}\text { 2. dificuldades para se orientar em } \\
\text { lugares novos }\end{array}$ & 43,3 & 60,0 & 56,1 & 0,09 & 0,167 \\
\hline $\begin{array}{l}\text { 3. dificuldades para se recordar de } \\
\text { ligações telefônicas } * 2\end{array}$ & 67,9 & 47,6 & 44,8 & 0,05 & 0,267 \\
\hline 4. dificuldades para entender conversas & 36,7 & 55,6 & 54,0 & 0,03 & 0,403 \\
\hline 5. dificuldades para escrever assinatura*4 & 26,9 & 69,2 & 55,2 & 0,31 & 0,021 \\
\hline $\begin{array}{l}\text { 6. dificuldades para entender o que } \\
\text { lê no jornal }{ }^{* 8}\end{array}$ & 40,9 & 55,5 & 56,1 & $-0,03$ & 0,583 \\
\hline $\begin{array}{l}\text { 7. dificuldades para guardar objetos } \\
\text { pessoais em ordem*1 }\end{array}$ & 51,7 & 41,4 & 50,4 & $-0,18$ & 0,929 \\
\hline $\begin{array}{l}\text { 8. dificuldades para lembrar onde deixa } \\
\text { as coisas em casa }\end{array}$ & 56,7 & 52,2 & 54,0 & $-0,04$ & 0,638 \\
\hline $\begin{array}{l}\text { 9. dificuldades para escrever } \\
\text { anotações ou cartas*5 }\end{array}$ & 52,0 & 69,0 & 57,5 & 0,27 & 0,012 \\
\hline 10. dificuldades para lidar com dinheiro & 40,0 & 60,0 & 48,0 & 0,23 & 0,044 \\
\hline $\begin{array}{l}\text { 11. dificuldades para se orientar } \\
\text { na vizinhança }\end{array}$ & 46,7 & 73,3 & 68,0 & 0,17 & 0,093 \\
\hline $\begin{array}{l}\text { 12. dificuldades para se lembrar } \\
\text { de compromissos }\end{array}$ & 63,3 & 44,4 & 46,1 & $-0,03$ & 0,614 \\
\hline $\begin{array}{l}\text { 13. dificuldades para praticar hobbies/ } \\
\text { passatempos favoritos }\end{array}$ & 36,7 & 58,9 & 51,6 & 0,15 & 0,145 \\
\hline $\begin{array}{l}\text { 14. dificuldades para se comunicar } \\
\text { com as pessoas }\end{array}$ & 33,3 & 67,8 & 60,7 & 0,18 & 0,080 \\
\hline 15. dificuldades para fazer cálculos mentais $* 6$ & 50,0 & 65,3 & 52,5 & 0,27 & 0,016 \\
\hline $\begin{array}{l}\text { 16. dificuldades para se lembrar das coisas que } \\
\text { tem que comprar quando vai às compras } * 2\end{array}$ & 42,9 & 70,2 & 55,6 & 0,33 & 0,006 \\
\hline $\begin{array}{l}\text { 17. dificuldades para controlar esfíncteres } \\
\text { (urina e fezes) }\end{array}$ & 20,0 & 84,4 & 78,7 & 0,27 & 0,023 \\
\hline $\begin{array}{l}\text { 18. dificuldades para entender o } \\
\text { enredo de um filme }\end{array}$ & 53,3 & 46,7 & 49,2 & $-0,05$ & 0,650 \\
\hline 19. dificuldades para se orientar em sua casa & 13,3 & 87,8 & 84,9 & 0,19 & 0,055 \\
\hline $\begin{array}{l}\text { 20. dificuldades para realizar atividades } \\
\text { da casa (cozinhar, limpar, consertar coisas) }\end{array}$ & 43,3 & 65,6 & 50,3 & 0,30 & 0,007 \\
\hline $\begin{array}{l}\text { 21. dificuldades para se alimentar? } \\
\text { (excluir inapetência)*1 }\end{array}$ & 10,3 & 88,5 & 83,7 & 0,30 & 0,016 \\
\hline $\begin{array}{l}\text { 22. dificuldades para utilizar talões de cheque } \\
\text { ou realizar pagamentos*3 }\end{array}$ & 40,7 & 51,8 & 52,5 & $-0,01$ & 0,534 \\
\hline 23. dificuldades para se vestir sem ajuda & 13,3 & 91,1 & 85,6 & 0,38 & 0,002 \\
\hline 24. dificuldades para tomar banho sem ajuda & 16,7 & 86,7 & 81,7 & 0,27 & 0,020 \\
\hline
\end{tabular}

*n, número de pares não disponíveis para análise. 
Tabela 3. Concordância dos escores entre pacientes e cuidadores para os itens do questionário de demênciaseção B: comportamento.

\begin{tabular}{|c|c|c|c|c|c|}
\hline Item do Questionário & $\begin{array}{l}\text { de pacientes } \\
\text { escore menor } \\
\text { o do cuidador }\end{array}$ & $\begin{array}{c}\text { Concordânica } \\
\text { observada } \\
(\%)\end{array}$ & $\begin{array}{c}\text { Concordância } \\
\text { esperada } \\
(\%)\end{array}$ & $\begin{array}{l}\text { Kappa } \\
\text { ponderadc }\end{array}$ & p \\
\hline $\begin{array}{l}\text { 1. mais rígido em decisões, menor } \\
\text { capacidade para se adaptar a novas situações*1 }\end{array}$ & $* 1$ & 59,8 & 49,7 & 0,20 & 0,089 \\
\hline $\begin{array}{l}\text { 2. mais egoísta, prestando menos atenção } \\
\text { nas necessidades das outras pessoas*1 }\end{array}$ & 34,5 & 54,0 & 58,1 & $-0,10$ & 0,731 \\
\hline $\begin{array}{l}\text { 3. mais irritado, perde paciência } \\
\text { com facilidade }\end{array}$ & 33,3 & 68,9 & 51,6 & 0,36 & 0,008 \\
\hline 4. crises de choro & 20,0 & 85,6 & 56,1 & 0,67 & $<0,001$ \\
\hline 5. ri em situações inadequadas*1 & 24,1 & 82,8 & 33,3 & $-0,03$ & 0,596 \\
\hline $\begin{array}{l}\text { 6. mais interessado em temas sexuais, } \\
\text { falando ou lendo sobre sexo }\end{array}$ & 3,3 & 91,7 & 92,0 & $-0,04$ & 0,601 \\
\hline $\begin{array}{l}\text { 7. perdeu o interesse em hobbies/passatempos } \\
\text { ou atividades que antes lhe interessavam }\end{array}$ & 40,0 & 45,6 & 49,3 & $-0,07$ & 0,681 \\
\hline 8. sente-se mais deprimido & 40,0 & 63,3 & 53,3 & 0,21 & 0,064 \\
\hline $\begin{array}{l}\text { 9. sente que alguém quer prejudicá-lo/a } \\
\text { (ou outros delírios)*1 }\end{array}$ & 27,6 & 69,0 & 65,9 & 0,09 & 0,271 \\
\hline $\begin{array}{l}\text { 10. ouve vozes, ou vê coisas que outras } \\
\text { pessoas não conseguem }\end{array}$ & 30,0 & 76,7 & 70,4 & 0,21 & 0,030 \\
\hline $\begin{array}{l}\text { 11. grita, xinga ou fica exaltado/a } \\
\text { com os outros }\end{array}$ & 23,3 & 74,4 & 69,3 & 0,30 & 0,016 \\
\hline 12. agride fisicamente os outros & 26,7 & 85,6 & 85,6 & 0 & 一 \\
\hline 13. problemas com sono & 36,7 & 76,7 & 58,3 & 0,44 & $<0,001$ \\
\hline 14. mais tenso ou aflito & 33,3 & 63,3 & 55,2 & 0,18 & 0,097 \\
\hline $\begin{array}{l}\text { 15. evitando situações específicas } \\
\text { por sentir medo }\end{array}$ & 33,3 & 66,7 & 63,6 & 0,08 & 0,276 \\
\hline 16. muito eufórico/a ou com muita energia & 20,0 & 78,9 & 72,5 & 0,23 & 0,041 \\
\hline $\begin{array}{l}\text { 17. comida em excesso ou de } \\
\text { forma descontrolada }\end{array}$ & 20,0 & 81,1 & 79,8 & 0,06 & 0,310 \\
\hline 18. perdeu apetite & 6,7 & 86,7 & 72,3 & 0,52 & $<0,001$ \\
\hline
\end{tabular}

*n, número de pares não disponíveis para análise.

variou de 53 a 89 anos e a dos cuidadores de 28 a 82 anos. Praticamente $3 / 4$ dos cuidadores eram do sexo feminino. Não havia pessoas sem alfabetização entre os cuidadores, mas 23,3\% dos pacientes não receberam educação formal. A renda familiar média variou de 0,5 a 33 salários mínimos para os pacientes e de 1 a 33 salários mínimos para os cuidadores.

A média dos escores no MMSE entre os pacientes foi 14,93 (IC=12,68 a 17,18; variação de 3 a 24) e entre os cuidadores 27,03 ( $\mathrm{IC}=26,22$ a 27,84; variação de 23 a 30). A gravidade do quadro demencial foi determinada de acordo com os escores no MMSE: 34,5\% apresentavam demência leve, $12,5 \%$ moderada, e $20,7 \%$ grave.

As Tabelas 2 e 3 apresentam a concordância entre pacientes e cuidadores nos escores individuais do QD. O valor do Kappa ponderado observado para os itens individuais do QD foi baixo para a maioria dos itens. O valor do índice de Kappa foi superior a 0,40 (indicando concordância moderada) em apenas 3 das questões que avaliavam comportamento: crises de choro, problemas com sono e perda do apetite. Para os demais sintomas, a concordância entre os escores foi baixa ou insignificante. 
O escore total para o QD-A foi calculado a partir da soma dos 24 itens individuais da escala. O escore total foi de 20,24 (IC=14,10 a 26,38) e 37,00 (IC=30,34 a 43,66) de acordo com pacientes e cuidadores respectivamente. O teste t-pareado revelou que o escore do QD-A de acordo com o paciente era significativamente menor do que o relatado pelo cuidador (t-pareado $=-4,07 ; \mathrm{p}<0,001$ — 9 pares perdidos para análise). De forma semelhante, o escore total para o QD-B foi 12,93 (IC=9,66 a 16,19) e 17,85 (IC=13,97 a 21,73) de acordo com pacientes e controles respectivamente (t-pareado= $-2,27, \mathrm{p}=0,032-3$ pares perdidos para análise).

A correlação de Spearman entre o escore no QD-A e o MMSE conforme avaliação do paciente foi -0,39 ( $\mathrm{p}=0,061$ ). De forma semelhante, a correlação entre o QD-A de acordo com a avaliação do cuidador e o escore no MMSE do paciente foi $-0,57(\mathrm{p}=0,007)$. A correlação entre o MMSE do paciente e o escore total no QD-B de acordo com o paciente (rho= $-0,22, \mathrm{p}=0,271$ ) e o cuidador $(\mathrm{rho}=-0,21, \mathrm{p}=0,266)$ foi baixa. A correlação entre o QD-A e QD-B de acordo com o paciente foi $0,47$ ( $\mathrm{p}=0,028)$. A correlação de Spearman entre os escores do MMSE e a diferença entre os escores do QD-A de acordo com os cuidadores e pacientes (anosognosia cognitiva) foi baixa (rho= $-0,14$, $\mathrm{p}=0$,554). De forma semelhante, a correlação de Spearman entre os escores do MMSE e a diferença entre os escores da seção B de acordo com os cuidadores e pacientes (anosognosia comportamental) não foi significativa (rho= $0,06, p=0,751)$. A correlação entre anosognosia cognitiva e comportamental foi insignificante $(\mathrm{rho}=0,02, \mathrm{p}=0,947)$.

Dezesseis cuidadores relataram que os pacientes sob seus cuidados estavam consistentemente deprimidos (escore $=3$ no QD-seção B, ítem 8). O escore total desses pacientes no QD-A, de acordo com o cuidador, foi 42,80 ( $\mathrm{IC}=33,24$ a 52,36 ), enquanto pacientes sem sintomas depressivos consistentes apresentaram escore total de 31,73 ( $\mathrm{IC}=22,15$ a 41,30; $\mathrm{t}=-1,83, \mathrm{p}=0,083)$. Pacientes com (média $=18,50, \mathrm{IC}=3,86$ a 33,14) e sem depressão (média= 15,18, IC= 3,01 a 27,35) apresentavam escores de anosognosia cognitiva semelhantes $(t=-0,40, p=0,698)$.

\section{DISCUSSÃO}

O diagnóstico da doença de Alzheimer baseia-se no uso de dados que indiquem a presença de deterioração da capacidade intelectual e funcional do indivíduo. Essas informações podem ser obtidas através de avaliação direta do paciente ou a partir de entrevista com o cuidador. É fundamental, porém, que as informações que servem de base para o diagnóstico sejam confiáveis. Há evidências, por exemplo, de que pacientes com DA têm consciência limitada da gravidade dos déficits cognitivos associados à doença ${ }^{13} \mathrm{e}$, portanto, fornecem informações pouco precisas sobre seu estado clínico. Assim, é importante obter informações sobre a condição clínica do paciente com seu cuidador. Mackinnon e Mulligan ${ }^{14}$ mostraram que a precisão do diagnóstico de 106 pacientes com demência aumentava quando, além do MMSE, se utilizavam também as informações obtidas com o cuidador.

Os resultados deste estudo mostram que pacientes com DA atribuem a si mesmo escores consistementente menores no QD-A que seus cuidadores. Há duas formas de explicar esse achado: pacientes subestimam suas dificuldades intelectuais e em atividades da vida diária, ou cuidadores super-estimam os déficits dos pacientes. $\mathrm{O}$ índice de correlação moderadamete elevado observado entre o escore do paciente no MMSE e o escore no QD-A de acordo com o cuidador $(\mathrm{rho}=-0,57)$ indica que o informante avalia de forma relativamente precisa a gravidade das dificuldades intelectuais do paciente. Em contraste, o índice de correlação entre o escore do paciente no MMSE e sua autoavaliação no QD-A (rho= -0,39) sugere que pacientes com DA tendem a subestimar suas limitações intelectuais. Neri et al. ${ }^{15}$ relataram achados semelhantes em sua avaliação de 80 pacientes com demência entrevistados com o 'Cambridge Examination for Mental Disorders of the Elderly' (CAMDEX). Naquele estudo, os autores observaram que não havia correlação significativa entre os escores do CAMCOG (a escala para avaliação cognitiva do CAMDEX) e auto-avaliação da memória dos pacientes. Por outro lado, os escores do CAMCOG se correlacionaram de forma significativa com a avaliação do informante. Assim, os dados deste e outros estudos ${ }^{6,15}$ indicam que pacientes 
com demência subestimam suas dificuldades intelectuais e que a entrevista com o cuidador pode fornecer informações mais precisas quanto às reais habilidades do paciente.

Os pacientes apresentaram, também, escores menores do que os cuidadores na seção do questionário que avalia psicopatologia (seção B), sugerindo que indivíduos com DA podem subestimar as alterações de comportamento por eles vivenciada. Apesar disso, os pacientes demonstraram capacidade relativamente preservada para identificar a presença de sintomas como irritabilidade, choro, depressão, e problemas com o sono ou apetite. Sete dos trinta pacientes incluídos no estudo apresentavam agressividade física de acordo com o cuidador — todos eles negaram ser fisicamente agressivos. Observamos, ainda, uma correlação não significativa entre o escore total do QD-B (para o paciente e cuidador) e o escore do paciente no MMSE. Esse resultado sugere que as alterações de comportamento na DA não estão diretamente associadas à gravidade do declínio cognitivo.

Pacientes que avaliaram suas dificuldades intelectuais de forma crítica relataram, também, mais problemas de comportamento (correlação de Spearman entre os escores $=0,47$ ). Há três possiveis explicações para este achado: (1) pacientes com boa auto-percepção são capazes de monitorar de forma adequada tanto as dificuldades cognitivas quanto comportamentais, (2) pacientes que se queixam de problemas comportamentais queixam-se também de dificuldades intelectuais ou, (3) pacientes que negam problemas comportamentais negam, da mesma forma, problemas intelectuais. Não foi possível investigar essas possibilidades de forma mais detalhada devido ao número limitado de participantes em nosso estudo, embora a experiência clínica sugira que pacientes com DA tendem a minimizar os déficits cognitivos e alterações comportamentais.

Os fatores que contribuem para comprometer a capacidade de autopercepção de pacientes com DA ainda não foram devidamente esclarecidos. Seltzer et al. ${ }^{16}$ observaram que a ausência de percepção quanto à existência de problemas com a memória entre 36 pacientes com DA estava associada à gravidade do quadro demencial. Sevush ${ }^{17}$ também observou associação entre a gravidade da demência e a falta de crítica quanto às dificuldades mnésticas do paciente, embora ele fosse incapaz de demonstrar que a falta de crítica em 106 pacientes com DA progredisse com a evolução da doença. Nós observamos, neste estudo, a existência de correlação negativa entre os escores do QD-A e MMSE - isto é, entre a percepção dos déficits intelectuais e a gravidade do declínio cognitivo. Entretanto, a correlação entre os escores de anosognosia cognitiva e MMSE foi baixa, sugerindo que a gravidade do quadro demencial influencia de forma limitada o desenvolvimento de anosognosia.

Seltzer et al. ${ }^{16}$ propuseram que, além da gravidade da demência, a presença de sintomas depressivos poderia contribuir para reduzir a capacidade autocrítica de pacientes com DA. Nossos resultados indicam que pacientes com sintomas depressivos tendem a apresentar maiores dificuldades intelectuais de acordo com a avaliação dos cuidadores, embora nossa análise revele, também, que a anosognosia desses casos não pode ser atribuída à presença de depressão. Reed et al. ${ }^{18}$ sugeriram que anosognosia estava associada a uma redução signficativa da perfusão sanguínea em região dorsolateral direita do lobo frontal de pacientes com DA. Este achado sugere que a dificuldade de auto-monitoramento observada entre esses pacientes indica o comprometimento de funções do lobo frontal (veja também Michon et al. ${ }^{19}$ e Ott et al. ${ }^{13}$ ) e não a presença de depressão ou gravidade do quadro demencial.

Os achados deste e outros estudos ${ }^{20}$ sugerem, ainda, que anosognosia não ocorre de forma homogênea para todas as funções cognitivas: a percepção das dificuldades com a memória é comprometida de forma mais intensa. Da mesma forma, a falta de insight para alterações de comportamento e sintomas depressivos parece ser particularmente proeminente entre pacientes com DA. Finalmente, a baixa correlação entre anosognosia cognitiva e comportamental sugere que a falta de crítica de pacientes com DA não representa uma função unitária, e sim a deterioração no monitoramento de diversas atividades mentais relativamente independentes. 
Em resumo, os resultados deste estudo indicam que pacientes com DA tendem a subestimar seus déficits intelectuais e dificuldades comportamentais - isso ocorre de forma independente da gravidade do quadro demencial ou da presença de sintomas depressivos. É fundamental, portanto, que profissionais de saúde obtenham sempre informações clínicas adicionais com o cuidador a fim de aumentar a sensibilidade do diagnóstico e otimizar o manejo clínico de pacientes com DA.

Agradecimentos - Agradecemos aos Drs. Regiane Garrido e Sérgio Tamai pelo estímulo e colaboração com recrutamento de pacientes.

\section{REFERENCES}

1. Brookmeyer R, Gray S, Kawas C. Projections of Alzheimer's disease in the United States and the public health impact of delaying disease onset. Am J Public Health 1998;88:1337-1342.

2. Henderson AS, Jorm AF. Dementia in Australia. Aged and Community Care Service Development and Evaluation Reports, No. 35, 1998. Canberra: Commonwealth of Australia.

3. Ebly EM, Parhad IM, Hogan DB, Fung TS. Prevalence and types of dementia in the very old: results from the Canadian Study of Health and Aging. Neurology 1994;44:1593-1600.

4. Almeida OP. Instrumentos para a avaliação de pacientes com demência. Rev Psiquiatr Clín 1999;26:78-89.

5. Almeida OP. Mini exame do estado mental (MMSE) e o diagnóstico de demência. Arq Neuropsiquiatr 1998;56:605-612

6. Jorm AF. Assessment of cognitive impairment and dementia using informant report. Clin Psychol Rev 1996;16:51-73.

7. Almeida OP. Sintomas psiquiátricos entre pacientes com demência atendidos em um serviço ambulatorial. Arq Neuropsiquiatr 1999;57:937-943

8. Burns A, Jacoby R, Levy R. Psychiatric phenomena in Alzheimer's disease: III. Disorders of mood. Br J Psychiatry 1990;157:81-86.

9. American Psychiatric Association. Diagnostic and statistical manual of mental disorders: DSM-IV - 4.ed. Washington, DC: American Psychiatric Association, 1994.

10. Folstein MF, Folstein SE, McHugh PR. Mini-mental state: a practical method for grading the cognitive state of patients for the clinician. J Psychiatr Res 1975;12:189-198.

11. Migliorelli R, Teson A, Sabe L, et al. Anosognosia in Alzheimer's disease: a study of associated factors. J Neuropsychiatry Clin Neurosci 1995; 7:338-344.

12. StataCorp. Stata statistical software: release 6.0. College Station, TX: Stata Corporation, 1999.

13. Ott BR, Lafleche G, Whelihan WM, Buongiorno GW, Albert MS, Fogel BS. Impaired awareness of deficits in Alzheimer's disease. Alzheimer Dis Assoc Disord 1996;10:68-76.

14. Mackinnon A, Mulligan R. Combining cognitive testing and informant report to increase accuracy in screening for dementia. Am J Psychiatry 1998;155:1529-1535.

15. Neri M, Roth M, De Vreese LP, et al.. The validity of informant reports in assessing the severity of dementia: evidence from the CAMDEX interview. Dement Geriatr Cogn Disord 1998;9:56-62.

16. Seltzer B, Vasterling JJ, Hale MA, Khurana R. Unawareness of memory deficit in Alzheimer's disease: relation to mood and other disease variables. Neuropsychiatry Neuropsychol Behav Neurol 1995;8:176-181

17. Sevush S. Relationship between denial of memory deficit and dementia severity in Alzheimer disease. Neuropsychiatry Neuropsychol Behav Neurol 1999;12:88-94.

18. Reed BR, Jagust WJ, Coulter L. Anosognosia in Alzheimer's disease: relationship to depression, cognitive function, and cerebral perfusion. J Clin Exp Neuropsychol 1993;15:231-244.

19. Michon A, Deweer B, Pillon B, Agid Y, Dubois B. Relation of anosognosia to frontal lobe dysfunction in Alzheimer's disease. J Neurol Neurosurg Psychiatry 1994;57:805-809.

20. Vasterling JJ, Seltzer B, Foss JW, Vanderbrook V. Unawareness of deficit in Alzheimer's disease: domain-specific differences and disease correlates. Neuropsychiatry Neuropsychol Behav Neurol 1995;8:26-32. 